\title{
El populismo como lógica hegemónica: una mirada a la perspectiva teórica elaborada por Ernesto Laclau*
}

\author{
Álvaro Iván López Bayona*
}

\begin{abstract}
RESUMEN
Recibido: 3 de abril de 2014

En este artículo se presenta una reflexión acerca de la teoría del

Evaluado: 20 de abril de 2014 populismo elaborada por Ernesto Laclau, como una perspectiva Aceptado: 8 de mayo de 2014 analítica que permite restituir el carácter político del mismo. Para llevar a cabo esto, se rastrea y explica la importancia de categorías como hegemonía y articulación en el pensamiento de Laclau, su viraje en el análisis de la constitución de las identidades políticas, pasando del grupo a la demanda como unidad análisis y, por último, la centralidad del lenguaje y el discurso como elementos configurantes de la realidad social.
\end{abstract}

Palabras clave: Populismo, hegemonía, articulación, discurso.

* Artículo de reflexión. Este artículo recoge algunos de los postulados centrales de la teoría del populismo elaborada por Ernesto Laclau, como una alternativa para el análisis de la constitución de las identificaciones políticas. Cómo citar este artículo: López Bayona, A.I. (2014). El populismo como lógica hegemónica: una mirada a la perspectiva teórica elaborada por Ernesto Laclau. Hallazgos, 11 (22), pp. 245-262.

** Sociólogo, Magster en Estudios Culturales, Universidad de los Andes. Docente Facultad Sociología Universidad Santo Tomás (Colombia). E-mail: alvarolopez@usantotomas.edu.co 


\title{
Populism as a Hegemonic Logic:
}

\section{A look at the theory and viewpoints as developed by Ernesto Laclau}

\begin{abstract}
This article presents a reflection about the theory of populism developed by Ernesto Laclau, as an analytical perspective allowing the restoration of the political nature thereof. For the foregoing to be carried out, the importance of categories such as hegemony and articulation, its turn regarding the analysis of the constitution of the political identities, going from the group to the demand as an analysis unit are tracked and explained the in the Laclau thought; and finally, the language centrality and discourse as elements configuring the social reality.
\end{abstract}

Keywords: Populism, hegemony, articulation, discourse. 


\section{O populismo como a lógica hegemônica:}

\section{um olhar sobre a perspectiva teórica desenvolvida por Ernesto Laclau}

\section{Resumo}

Neste artigo apresenta-se uma reflexão sobre a teoria do populismo desenvolvida por Ernesto Laclau, como uma perspectiva analítica que permite restaurar a natureza política do que está-se apresentado. Para conseguir isso, se rasteja e explica a importância de categorias como hegemonia e articulação no pensamento de Laclau, sua viragem na análise da constituição das identidades políticas, passando do grupo para à demanda como unidade de análise e, finalmente, a centralidade da linguagem e do discurso como elementos configurantes da realidade social.

Palavras-chave: Populismo, hegemonia, articulação, discurso. 


\section{INTRODUCCIÓN}

Un término que ha sido objeto de uso y abuso por parte de las ciencias sociales es el de populismo. Su uso más común y corriente ha estado orientado a describir y explicar las movilizaciones populares como movimientos irracionales, vagos e indeterminados donde las masas -la plebs - son manipuladas por un líder carismático o un grupo social en contra de los "ricos" o "privilegiados". Esta es la perspectiva racionalista. Otro acercamiento, más asociado a una perspectiva reinvindicativa, pero con un fuerte sesgo de clase, ha explicado el populismo como un movimiento de carácter popular reinvindicativo marcado por su ascendencia de clase a través del cual se pretende implementar una forma de gobierno redistributiva que favorezca al pueblo - proletario-. En este artículo se presenta un acercamiento diferente a estas dos perspectivas, tomando como punto de referencia el enfoque teórico elaborado por Ernesto Laclau (2005).

En esta medida, nos apartamos tanto de la perspectiva racionalista como de la reinvindicativa reducida a su carácter de clase, las cuales restringen el populismo a una lógica irracional, o una simple movilización popular con carácter clasista, para entenderlo como una lógica hegemónica por medio de la cual se produce un pueblo - un populus como resultado de una producción discursiva, operación a través de la cual este se constituye como el lugar de cristalización o centro articulador de un conjunto de demandas, grupos y sectores sociales diferentes entre sí.

Para cumplir con este propósito, se realiza una reconstrucción de tres elementos centrales desarrollados por Laclau (1977; 1985; 2005; 2005a) dentro de su perspectiva analítica. En primer lugar, el empleo de la hegemonía y la articulación como categorías de análisis fundamentales, para rastrear la constitución de las identidades o identificaciones políticas. En segundo lugar, el cambio en la unidad de análisis pasando del grupo a la demanda. Y, por último, la importancia atribuida al lenguaje y, más específicamente, al discurso como una práctica política a través de la cual se organiza y clasifica el mundo social de forma contingente, es decir, de forma figurativa y no literal.

\section{LA HEGEMONÍA Y LA ARTICULACIÓN: LA DES-ESENCIALIZACIÓN DE LO SOCIAL}

Para comenzar, es importante recordar que el interés de Laclau (2005) por el análisis del populismo se enmarca en una discusión con una corriente de pensamiento racionalista, representada por teóricos sociales como Gino Germani, Margaret Canovan, Ghita Ionescu y Ernest Gellner, la cual, a través de la asociación teórica e histórica de este a una forma incoherente, irracional y vaga de movilización social, lo confinó a una "posición marginal en el discurso de las ciencias sociales" (p. 34).

El resultado de este confinamiento, como lo señala Laclau, fue la asimilación del populismo como "el simple opuesto de formas políticas dignificadas con el estatus de una verdadera racionalidad" (p. 34), y la caracterización del mismo como un "fenómeno político aberrante" (p. 35) que impedía el tránsito de la sociedad a formas cada vez más racionales de organización política. Frente a esta interpretación "peyorativa" 
del populismo, Laclau presenta otra forma de abordarlo e interpretarlo que consiste en entenderlo como una lógica política que presenta "un determinado modo de articulación" de una serie de "contenidos sociales, políticos e ideológicos" (2005a, p. 53), diferentes entre sí, articulados no por una coincidencia ideológica o sociológica entre los mismos - necesaria correspondencia-, sino por una práctica hegemónica a través de la cual se articulan "diferentes demandas fragmentadas y dislocadas en torno a un nuevo centro" (2005, p. 222). Este nuevo centro es lo que Laclau denomina significante vacío, categoría que será explicada más adelante.

Ahora bien, en cuanto a la hegemonía y la articulación, cabe señalar que estas dos categorías permitieron a Laclau realizar dos cosas en particular. En primer lugar, romper con la concepción de la historia como "un avance continuo infinito" (p. 281) hacia formas de organización social cada vez más racionales. Y en segundo lugar, realizar una ruptura con una concepción marxista ortodoxa a través de la cual se restringía la conformación de los grupos sociales a la necesaria correspondencia entre los intereses ideológicos y la posición de clase ocupada por los sujetos dentro del modo de producción capitalista. Detengámonos por un momento, para explicar el primer aspecto: la historicidad de la historia o, en otras palabras, la producción de esta como una práctica social.

La concepción de la historia como un recorrido racional, decretado de manera teleológica y transparente por una racionalidad absoluta, que determina la forma cómo esta debe desenvolverse en sucesivas etapas históricas, por ejemplo, esclavismo, feudalismo, capitalismo, socialismo y comunismo, las cuales deben conducir a formas de organización social cada vez más racionales, es desvirtuada por Laclau gracias al empleo de la hegemonía como categoría de análisis en el terreno político. Para Laclau (1985), la hegemonía no es nada más que una categoría que se nos presenta como una salida a la idea de una "necesidad histórica" producida y reproducida por el marxismo clásico para explicar el desarrollo de la historia (p. 27). En este sentido la hegemonía, en términos conceptuales como operativos, debe ser entendida como un "juego [político] que no es nunca 'suma cero' porque las reglas y los jugadores no llegan a ser jamás plenamente explícitos" (p. 239), como se pretendía en la concepción marxista ortodoxa. Con esta operación se deja de lado la concepción de la política y de las luchas políticas como algo determinado y determinante, para ser entendidas como algo en constante disputa y redefinición por las condiciones históricas en las cuales se llevan a cabo y por los sujetos involucrados en ellas.

La consecuencia inmediata de esta visión para el análisis político es el abandono de la concepción de la historia y, por lo tanto, de las relaciones sociales que se constituyen en y a través de ella como la secuencia y el efecto lógico y racional de una teodicea universalista que determina la marcha de estas de forma ordenada y transparente. De ahora en adelante, gracias al empleo de la hegemonía, la historia y las prácticas sociales son entendidas como confrontaciones hegemónicas, resultado de luchas políticas concretas ubicadas socio-históricamente entre diferentes grupos sociales en las cuales no existe garantía alguna de lo que pueda llegar a suceder. 
Así pues, la historia y las relaciones sociales dejan de ser entendidas como lógicas teleológicas y comienzan a ser entendidas como "una sucesión discontinua de formaciones hegemónicas que no puede[n] ser ordenada[s] de acuerdo con ninguna narrativa universal que trascienda su historicidad contingente" (2005, p. 281). Con esta nueva forma de concebir la historia y las relaciones sociales, el esclavismo, el feudalismo y el capitalismo, por ejemplo, corresponderían a formaciones hegemónicas más que a etapas sucesivas y lineales determinadas por una racionalidad absoluta.

En relación al segundo aspecto, la ruptura con una concepción marxista ortodoxa de clase, es la articulación la que juega un papel central aquí. La articulación, al ser entendida como una forma de conexión a través de la cual se crea una unidad de dos o más elementos diferentes bajo determinadas condiciones históricas (Hall, 2010), permite ir más allá de la idea de un grupo conformado únicamente por intereses de clase o sectoriales. Dicho de otra manera, la articulación permite pensar en grupos sociales articulados por una diversidad de sectores, que no guardan una relación necesaria entre sus "elementos ideológicos y su pertenencia de clase" (p. 85), sino que son el resultado de un nuevo proceso de identificación.

Con esta reorientación epistemológica, lo que se pone en cuestión es la noción de sujeto unitario cartesiano y la identidad como algo establecido y definido de una vez y para siempre. Por ejemplo, un movimiento ecologista y un "nuevo" sujeto ecológico desde esta perspectiva es el resultado de defender demandas tan diferentes como la conservación del medio ambiente, el aumento salarial de los trabajadores de un banco estatal, una reivindicación racial, la defensa del matrimonio igualitario entre personas del mismo sexo, entre muchas otras demandas. De esta manera, el nuevo sujeto popular ecológico es el resultado de una articulación de todas las anteriores demandas en una demanda popular, como lo puede ser la defensa de los derechos humanos y de un nuevo proceso de identificación social, no de una identidad o sujeto previo.

Esta nueva mirada dela historia y la conformación de los grupos sociales como producto de prácticas hegemónicas, abre el camino para el análisis del populismo desde otro punto de vista con dos consecuencias fundamentales. Por un lado, el abandono de la concepción racionalista del pueblo como una forma irracional y vaga de movilización social (perspectiva racionalista) y, por otro lado, la pérdida de validez de la perspectiva que asumía al mismo como la manifestación de un sujeto universal, homogéneo, constituido a priori y con una tarea teleológicamente determinada (perspectiva marxista ortodoxa). El efecto inmediato de esta reorientación en la interpretación del populismo es la comprensión del mismo como el resultado de una práctica articulatoria a través de la cual se articulan "una pluralidad de demandas democráticas" (Laclau, 2005a, p. 57) y grupos sociales diferentes entre sí en torno a un ideal u objetivo colectivo.

Otro resultado de esta nueva mirada desencializadora de lo social es que el pueblo pasa a ser entendido como "un modo de identificación a disposición de cualquier actor político" (Panizza, 2005, p. 14) que se encuentre inmerso en una lucha hegemónica, y no como un referente fijo al cual deben 
adherirse ciertas demandas y ciertos sujetos sociales. En este sentido, el pueblo pierde su connotación "esencial" de ser un lugar reservado únicamente para las reivindicaciones de los más pobres, los más oprimidos o los más explotados, para pasar a ser una de las formas discursivas a través de las cuales se intenta construir un proceso de identificación.

Llegados a este punto, podemos aventurarnos a realizarla siguiente conclusión de acuerdo con Laclau (2005). El pueblo como lugar de cristalización de una nueva subjetividad política "no está formado necesariamente por los pobres y tiene poco que ver con las nociones marxistas de alianza de clases contra la clase económicamente dominante" (p. 31); por el contrario, es el resultado de una "construcción discursiva contingente" (p. 117), donde no existe garantía alguna de qué grupos o demandas lo vayan a estructurar. Como nos lo recuerda Reyes (2005), el pueblo es el resultado de un proceso de nominación por medio del cual se "determina de manera retroactiva el sentido" (p. 143) del mismo y no de una necesidad histórica. Así, el pueblo como sujeto popular es el resultado de una producción discursiva por medio de la cual se determinada de manera contingente y no literal el "significado" que llega a adquirir y transmitir él mismo.

Continuando con esta línea argumentativa, es importante realizar la siguiente aclaración con el fin de evitar una lectura "relativista" de la constitución del pueblo a través de la cual se asuma que este puede llegar a significar cualquier cosa, desconociendo de esta manera, las condiciones históricas particulares donde él mismo se constituye.
Por ejemplo, no es lo mismo hablar de un pueblo constituido como resultado de una lucha hegemónica, donde el grupo que logra asumir el liderazgo como grupo medular dentro de la sociedad se identifica con una ideología de derecha, que uno constituido por un grupo cercano a una ideología de izquierda, o por uno de centro-derecha que por uno de centro-izquierda. Por otra parte, no va a ser lo mismo un pueblo constituido durante los años cuarenta del siglo XX en la sociedad colombiana, por ejemplo, que uno constituido durante la primera década del siglo XXI. De hecho, a partir de estas distinciones analíticas e históricas, se puede señalar que, aunque "el pueblo no posee un referente fijo, ni un significado esencial, lo cual equivale a coincidir [...] que el término significa lo que aquellos que lo utilizan eligen que signifique" (Panizza, 2005, p. 16), este, por más indeterminación del lenguaje, no es el resultado de la voluntad de los individuos ni de un determinismo a histórico. Por el contrario, es el resultado de una lucha hegemónica a través de la cual se instituye el sentido del mismo. En otras palabras, el pueblo, por más que no posea un referente fijo ni estable, se circunscribe de acuerdo a unas determinadas condiciones históricas, sociales, políticas, culturales e ideológicas, a una definición del mismo como la definición válida y legítima, es decir, como la hegemónica.

Teniendo como punto de referencia la anterior reflexión, podemos sostener la siguiente hipótesis en cuanto al populismo como una lógica política, esto es, hegemónica, a través de la cual se construye al pueblo: al ser el populismo una de las dimensiones fundamentales de lo político, a través de la cual se "construye y otorga significado al "pueblo" 
(Reyes, 2005, p. 134), este último no puede ser sino el resultado de una producción discursiva a través de la cual se pretende realizar un cierre arbitrario sobre el sentido del significante pueblo. Por lo tanto, una consecuencia fundamental de esta reformulación en términos epistemológicos es la inversión realizada en los términos del análisis político: es el populismo el que construye el pueblo y no a la inversa como se consideraba en la corriente ortodoxa del pensamiento marxista. Con esta des-esencialización del pueblo, pasamos al segundo elemento central en el cambio de enfoque realizado por Laclau, el paso del grupo como unidad de análisis a la demanda.

\section{DEL GRUPO A LA DEMANDA COMO UNIDAD DE ANÁLISIS}

Al no ser el pueblo el resultado de una esencia común subyacente compartida por los sectores y las demandas asociadas en torno a él, sino el resultado de una articulación hegemónica de las mismas en la cual no comparten nada positivo, solamente el hecho de que todas permanecen insatisfechas, tomar el grupo como unidad de análisis "oscurece" y "encubre" las múltiples particularidades que pueden llegar en un momento histórico particular a estructurarlo. Dicho de otra manera, la unidad del pueblo debe ser pensada como un resultado que esconde su mismo proceso de constitución, el cual, a través de un complejo proceso de articulación "de piezas diversas y divergentes que tienen la apariencia de [una] totalidad" (Grossberg, 2010, p. 100), lleva a cabo una operación de "totalización y opacización" que presenta a dicha "totalidad" como una unidad armónica y sin fisura alguna. Por lo tanto, el interés de Laclau se traslada al análisis de la articulación equivalencial de las demandas como factor fundamental en la constitución del pueblo.

Laclau (2005) puntualiza que el tipo de demandas que le interesa analizar son aquellas que realizan el tránsito de simples peticiones a reclamos con carácter de reivindicación, es decir, las que presentan un cierto desafío al sistema institucional para su resolución. Este desafío puede ser resuelto de manera administrativa cuando opera la lógica de la diferencia, o puede quedar suspendido, lo que abre la posibilidad para el surgimiento de una lógica populista. Este tipo de demandas son dos, las democráticas y las populares.

En cuanto al primer tipo de demandas, son las que se formulan "al sistema por alguien que ha sido excluido del mismo" (Laclau, 2005, p. 158), pero no se articulan equivalencialmente, es decir, permanecen aisladas. Estas demandas son fácilmente asimilables por el sistema, puesto que este las puede solucionar de manera aislada o administrativa. Este tipo de demandas pueden ser asociadas a lo que Laclau y Mouffe (1985) denominan como momentos $\mathrm{o}$, en otras palabras, a posiciones diferenciales dentro del discurso. Las demandas democráticas no presentan un serio desafío al orden social existente, pues su "exceso de sentido" es fácil y rápidamente clausurado por una lógica diferencial. Por ejemplo, cuando un grupo de estudiantes está reclamando mejor calidad en la educación, y otro grupo social, como el de los profesores, está reclamando mejores sueldos, y esto se da al mismo tiempo, pero estas demandas y sectores no se articulan y son resueltas de manera diferencial y administrativa por el sistema escolar, se clausura parcialmente 
el "exceso" de sentido de dichas demandas: la solución ofrecida por el sistema a las demandas elevadas por los estudiantes y profesores es mejorar la planta de docentes y subirles el sueldo a los últimos. En este caso se ha producido una lógica de la diferencia, anulando un potencial conflicto antagónico entre estudiantes y profesores contra el sistema escolar - directivos-.

El otro tipo de demandas son las populares, Laclau (2005) se refiere a ellas como "la pluralidad de demandas que, a través de su articulación equivalencial, constituyen una subjetividad más amplia" (p. 99) que las transciende en su particularidad. Esta subjetividad, para nuestro caso, está referida al pueblo.

Estas demandas, al estar articuladas en torno a una cadena equivalencial que las comienza a representar, ceden en cierto grado su condición particular a una demanda que las representa a todas ellas, dentro de un campo antagónico. Un ejemplo de esta forma de articulación es cuando diferentes grupos sociales, por ejemplo, los sindicalistas, los campesinos y los empresarios, teniendo cada uno de ellos diferentes demandas como las mejoras salariales para los primeros, el incentivo a la producción agrícola para los segundos y la apertura de nuevos mercados para los últimos, se articulan alrededor de una demanda como lo puede ser el desarrollo económico. Dicha demanda es la que comienza a significar toda la cadena equivalencial, significando algo más que su simple contenido particular. Esto quiere decir que esta demanda no sólo significa desarrollo económico sino también mejoras salariales, incentivo a la producción agrícola y apertura de nuevo mercados. El resultado de esta articulación equivalencial es la constitución de una nueva identidad colectiva que viene a representar a las demás identidades.

El resultado de este proceso de identificación es una articulación política o equivalencial, lo que quiere decir que se trata de un enlace temporal entre diferentes grupos y demandas, donde no se presenta una necesaria correspondencia ideológica entre las posiciones objetivas ocupadas por los sujetos sociales, la posición política que estos asumen en un momento histórico determinado y las demandas por las cuales estos se agrupan en una nueva identidad política. No obstante, un aspecto que debemos recordar de este tipo de articulación es que para que sea concebida como hegemónica, debe constituirse en y contra un cierto número de elementos que son percibidos como antagónicos, como el "enemigo" del pueblo. En este caso podríamos identificar, hipotéticamente, dichos elementos como un estado autoritario, regulador en lo económico y con poco empeño industrializador. Ilustremos esta dinámica a través del siguiente diagrama.

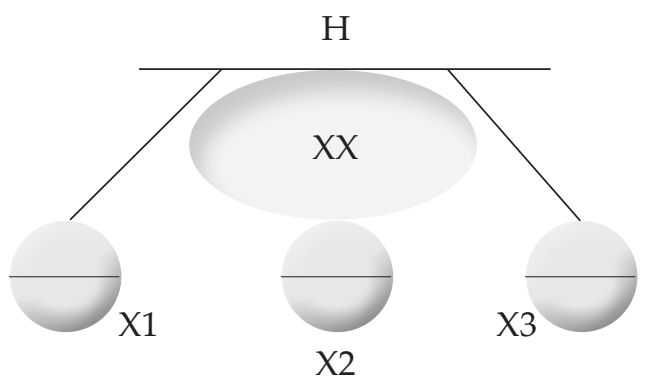

Fuente: elaboración propia 
Acá H representa el polo antagónico, o sea, el estado autoritario, regulador en lo económico y con poco empeño industrializador contra el cual las demandas X1 -mejoras salariales-, X2 -incentivo a la producción agrícola- y X3 -apertura de nuevos mercados- elevan sus reclamos; por su parte XX es la demanda particular que las articula -desarrollo económico-. De acuerdo a este diagrama y a la perspectiva de Laclau, XX se convierte en el significante vacío que logra articular a los significantes flotantes o demandas X1, X2 y X3, los cuales, a pesar de su articulación equivalencial, siguen manteniendo en cierto grado su condición de demandas particulares. Esta condición es la que se mantiene en la parte inferior de cada círculo.

Una consideración que debemos tener presente respecto al esquema anterior es que el significante que va "a adquirir el rol articulador va a depender, obviamente, de una historia contextual" (p. 114) y no de una determinación lógica o racionalista, lo que nos obliga a señalar que es el conjunto de condiciones histórico-sociales el que determina, en cierta medida, cuál demanda o elemento adquiere esa centralidad. Esta consideración tiene como consecuencia que el sentido de las demandas - significantes flotantesva a permanecer indeciso entre diferentes campos antagónicos hasta que alguno de los dos logré hegemonizar el campo social.

En este punto, es importante detenernos en un aspecto de particular interés dentro de la teoría del populismo elaborada por Laclau, ese trata dela relación existente entre las demandas populares, la construcción de las identidades políticas y el establecimiento de un antagonismo social.

\section{El ANTAGONISMO SOCIAL Y LA PRODUCCIÓN DE LA FRONTERA ANTAGÓNICA: EL PAPEL DE LOS SIGNIFICANTES VACÍOS}

Para comenzar, es importante señalar que el antagonismo es un elemento fundamental dentro de la lógica populista, puesto que es el que posibilita la construcción de una frontera antagónica entre un nosotros y un ellos, es decir, entre dos identidades políticas que se disputan la posibilidad de establecer su visión del mundo como la visión válida y legítima. Una de estas identidades la constituye la identidad popular, y, por tanto, "oprimida", mientras la otra es "la identidad negativa" que impide la plena realización de la primera.

Un aspecto muy significativo de este tipo de relación antagónica, es que esta "presupone la presencia de algunos significantes privilegiados que condensan en torno de sí mismos la significación de todo un campo antagónico" (p. 114) en contra de otro campo antagónico. Algunos de estos significantes pueden ser la oligarquía, la élite política, o el Estado, los cuales representan en un momento determinado al "enemigo", mientras los desposeídos, los humildes o los desfavorecidos representan al pueblo.

Esto significa que el antagonismo es una relación social a través de la cual se produce una división dicotómica del espacio social entre dos identidades políticas excluyentes, pero mutuamente dependientes: una que se instituye como la "buena" comunidad o los "buenos" ciudadanos, y otra que se establece como el "enemigo" de dicha comunidad. No obstante, es necesario aclarar que ninguno de estos significantes posee un privilegio 
apriorístico de inscripción, pues el pueblo, en un momento histórico particular, puede estar conformado por la oligarquía, los militares o la clase dirigente, así como el "enemigo" puede estarlo por los humildes, los desposeídos o el estado de bienestar. De hecho, esta configuración hegemónica fue la establecida durante la década de los ochenta, por políticos neoliberales como Margaret Thatcher en Gran Bretaña y Ronald Reagan en Estados Unidos, quienes señalaron a los pobres como los parásitos de la sociedad, y al estado de bienestar como un lastre para la economía liberal.

Los significantes que condensan todo un campo antagónico son denominados por Laclau como significantes vacíos, los cuales se fundan en "un ideal político universalizado que nunca puede ser satisfecho" (Lowndes, 2005, p. 205), porque su misma condición de apertura semántica impide su plena realización. En este sentido, los significantes vacíos no poseen una definición positiva, o conceptualmente estricta y precisable que los amarre a una lógica descriptivista o denotativa; por el contrario, están asociados a una lógica connotativa o antidescriptivista, en la cual la idea de la "emancipación del significante de cualquier dependencia del significado" (Laclau, 2005, p. 132) es central. Si no cumplieran con esta condición, no se podría hablar de una frontera antagónica como lo señala Laclau.

De allí que un significante vacío sea un significante que no se agota en una lógica denotativa, puesto que desborda la literalidad de los significados puramente conceptuales pudiendo ser un símbolo, una palabra o una imagen que comienza a perder su contenido particular para comenzar a significar algo más que sí mismo. Este algo más, es la significación de toda la cadena equivalencial que se encuentra compuesta por las diferentes demandas y sectores sociales.

Una función que se debe destacar de los significantes vacíos es que estos permiten "la comprensión de una cosa en términos de otra" (Jociles, 2005, p. 154), o la equivalencia de un término por otro. Por ejemplo, orden o seguridad dentro de un discurso populista, ya sea de derecha o izquierda, se pueden llegar a equivaler en un momento determinado a otro término, como lo puede ser la patria. Esta función la describe Laclau (2005) como la función catacrética del lenguaje, función a través de la cual se logra establecer "una relación de sustitución entre términos sobre la base del principio de analogía" (p. 34), teniendo como propósito estructurar las bases experienciales de las personas.

Teniendo como base las anteriores precisiones conceptuales, podemos aventurarnos a realizar una definición de los significantes vacíos, que nos permita clarificar su estatus epistemológico y su relación con la construcción de una frontera antagónica. Los significantes vacíos son una especie de "punto de condensación" (p. 226) entre diferentes "demandas que no comparten nada positivo, sólo el hecho de que todas ellas permanecen insatisfechas" (p. 125) respecto a las soluciones ofrecidas por el orden social existente, lo que se traduce en que los significantes vacíos no poseen una definición positiva o conceptualmente estricta que los amarre a una lógica descriptivista, pues su sentido no se circunscribe a este tipo de definiciones, sino a su capacidad de significar toda una cadena equivalencial. El resultado de esto, por lo tanto, es la constitución de 
una frontera política y de una nueva identidad política.

Otro aspecto importante de la relación entre las demandas populares, la construcción de identidades políticas y el establecimiento de un antagonismo social, es que son al mismo tiempo el resultado de una lógica articulatoria, pero también lo que posibilita que se mantenga, aunque sea de manera temporal. Expliquemos esta relación con mayor detalle.

Como lo señala Panizza (2005), al ser el populismo una lógica articulatoria, este necesariamente se constituye en un modo de identificación a través del cual no sólo se construye una frontera antagónica que divide el espacio social entre dos polos antagónicos - el pueblo vs. su enemigo-, sino también en un modo de identificación a través del cual se construyen identificaciones entre diferentes grupos sociales, estableciendo una articulación entre ellos. Esto significa que el populismo es una de las formas políticas de construir la unidad del grupo y su identidad, en contra de la de otros grupos.

En esta medida, la identidad del grupo es el resultado de un complejo proceso de articulación equivalencial entre diferentes demandas y sectores en un sistema variable de significación y no una esencia determinada por condiciones apriorísticas. Este proceso tiene dos momentos; uno contingente, donde no existe garantía de que las demandas y sectores se vayan a articular, pero sin embargo lo realizan a través de una lógica articulatoria; y otro de fijación parcial, donde las demandas se "estabilizan" temporalmente en un sistema variable de significación al compartir una condición de insatisfacción u hostilidad hacia algo o alguien. Estos dos momentos podrían ser denominados como el de la flotación y el de la fijación, sin querer decir con esto que uno de los dos prime sobre el otro. Como lo advierte el propio Laclau (2005), no existe un momento dominado exclusivamente por la flotación constante de los significados, lo cual equivale a sostener que nunca se podría establecer un cierto orden -significado- a las cosas, pero tampoco existe uno dominado completamente por el cierre absoluto de los significados, lo cual equivale a decir que todo orden es eterno. Por lo tanto, la conclusión es que todo orden es un intento realizado, más nunca plenamente logrado, por establecer un cierto tipo de orden, un cierre denotativo, en una realidad altamente contingente. El populismo opera bajo esta lógica articulatoria.

Un último aspecto que es necesario reseñar dentro de esta reflexión es la condición hegemónica que presentan las demandas populares y las identidades políticas populares. Como lo apunta Laclau, la aplicación de la hegemonía al análisis del populismo y de la construcción de identidades políticas, "tiene sentido sólo si la inscripción popular de demandas democráticas no procede de acuerdo con un diktat dado a priori o teleológicamente determinado, sino que es una operación contingente que puede moverse en una pluralidad de direcciones [ideológicas]" (p. 160). Esta pluralidad de direcciones puede corresponder a movimientos políticos de derecha, de izquierda, de liberales, de conservadores, de religiosos ortodoxos, de centro-izquierda, etc., lo que nos hace pensar que el populismo, como lógica política que constituye lo social, puede ser constituido, apropiado y reapropiado por fuerzas sociales tan diversas y divergentes como las anteriormente señaladas. 
Con relación a lo anterior, se puede sostener entonces que "ninguna demanda social tiene como su 'destino manifiesto' una forma a priori de inscripción, todo lo contrario [ésta] depende de una lucha hegemónica" (2005a, p. 63) por medio de la cual dichas demandas pueden ser reconfiguradas de diferentes maneras por parte de los grupos sociales que se encuentran inmersos dentro de la disputa hegemónica. De allí que el sentido que obtenga cierta demanda o elemento, dependa exclusivamente del sentido que le otorgue el grupo social que haya logrado constituirse como el grupo medular. Por ejemplo la democracia, el Estado, la seguridad, el orden, la economía, entre otros, no van a ser portadores del mismo sentido si el grupo social hegemónico que logra hegemonizar el espacio social y político, se encuentra cercano a un pensamiento de derecha o a uno de izquierda, lo cual equivale a decir que estos significantes, en este caso flotantes, cambian de significado dependiendo de la operación de cierre denotativo que se realice sobre ellos, y del lugar que ocupen dentro de la frontera antagónica.

Para cerrar este apartado y abrir paso al siguiente, es indispensable señalar tres consecuencias de lo dicho hasta el momento. En primer lugar, que al ser entendido el populismo como una lógica política a través de la cual se instituye lo social, este permite pensar en la construcción discursiva de un pueblo, un nosotros, en contra de un ellos (Reyes, 2005). Entonces el populismo permite pensar en la constitución antagónica de la sociedad. En segundo lugar, que el populismo, al estar referido a la constitución de una frontera antagónica a través de la cual se posibilita la "construcción discursiva del enemigo" (Laclau, 2005a, p. 59), este permite como herramienta analítica rastrear tanto la constitución de dicha frontera antagónica como la de las identidades políticas que se constituyen como las representantes legítimas de cada uno de los dos polos antagónicos constituidos por esta frontera. $\mathrm{Y}$ por último, que el populismo, al profundizar y resaltar la constitución política de la sociedad, es decir, la constitución conflictiva y contingente de la misma, permite emprender un camino analítico de la constitución de todo grupo social como un movimiento contingente e híbrido que alcanza su grado de "estructuración" gracias a la articulación de diferentes demandas y grupos sociales alrededor de una cadena equivalencial articulada por un significante vacío, el cual puede ser hegemonizado de manera diferente de acuerdo a que un grupo social logre hegemonizar el espacio social.

\section{LENGUAJE Y DISCURSO: LA NO LITERALIDAD DEL MUNDO SOCIAL}

Lo dicho hasta el momento nos abre el camino para hablar de la importancia del lenguaje y del discurso dentro de la teoría del populismo elaborada por Laclau. Esta importancia se encuentra referida a dos elementos en particular: la capacidad performativa del discurso y la facultad del lenguaje de subvertir los significados puramente denotativos.

Respecto a la capacidad performativa, como lo señalan Austin (1962) y Bourdieu (1999), está referida a la facultad del discurso de producir realidades a través de lo que se nombra, de crear performativamente una determinada realidad social. Sin embargo, para que la performatividad cumpla su propósito se deben cumplir y respetar ciertas condiciones sociales, por ejemplo, que quien pronuncie 
el discurso sea la persona autorizada para hacerlo, que las cosas que pronuncie sean las cosas legítimas de pronunciar, y que el lugar donde las pronuncie sea el lugar indicado para hacerlo. El cumplimiento de estas condiciones produce como resultado, que las palabras pronunciadas adquieran el estatus de "verdad", determinando la forma "correcta" y "adecuada" de acción y pensamiento para los sujetos sociales.

Por su parte, la subversión connotativa está referida a la imposibilidad de realizar un cierre denotativo de la producción del lenguaje; dicho de otra manera, de clausurar de forma definitiva el sentido que puedan adquirir las palabras dentro de los contextos en los cuales estas son utilizadas. En esta medida, la utilización de un cierto número de recursos retóricos (argumentos, metáforas, metonimias, sinécdoques, etcétera) por medio de los cuales se realizan operaciones asociativas entre las palabras, "desviándolas de su sentido literal" (Laclau, 2005, p. 96), es un elemento fundamental de toda práctica política.

Dentro de esta importancia atribuida al discurso y al lenguaje como productor de realidades sociales, es pertinente señalar la fuerza que Laclau le atribuye a la retórica como elemento constitutivo de lo social. Esta fuerza está referida, en primera medida, a su función catacrética, la cual permite realizar desplazamientos semánticos de términos o significados puramente denotativos, o significados figurativos. Es decir que a través de esta función se realiza un desplazamiento de una lógica denotativa del lenguaje, que amarra las palabras a un significado "original" $\mathrm{o}$ "esencial", a una lógica connotativa, donde las palabras comienzan a perder esa atadura "original", para comenzar a significar algo totalmente diferente de su significado "esencial". Con la operacionalización de esta función, la idea de identidades fijas y predeterminadas queda relegada y desplazada por la constitución contingente de las mismas.

Un segundo aspecto destacado por Laclau dentro de la performatividad y subversión del lenguaje, es la centralidad de la figura de la sinécdoque, figura literaria que permite establecer un tipo de relación particular entre la parte y el todo, donde la parte comienza a representar el todo, a constituirse como el todo. Esta es la función que cumple precisamente el significante vacío, el cual, podríamos aventurarnos a decir, es la sinécdoque por excelencia.

Por último, resalta la capacidad de la retórica para presentar como "verdadero", ante los potenciales receptores, lo que se anuncia a través del discurso. Esta capacidad de verosimilitud se asocia, como lo señala Perelman (1977), al uso de diferentes figuras literarias que tienen como finalidad persuadir y convencer a un determinado grupo de personas buscando su adhesión a las tesis expuestas por el orador (p. 12).

De acuerdo a lo anterior, podemos sostener que las dos primeras funciones están asociadas a la noción y el papel que desempañan los significantes vacíos y flotantes dentro del discurso; mientras que la última está asociada al estilo epidíctico de la retórica, estilo que tiene como propósito fundamental "intensificar la adhesión a [determinados] valores, teniendo como objetivo encontrar un punto de apoyo" (p. 41) universal para articular a diferentes grupos o sectores sociales, alrededor de un 
objetivo común o, desde nuestra perspectiva, alrededor de una demanda popular.

Las consecuencias de todo esto para la teoría del populismo son tres. Primero, el giro hacia la retórica como una operación discursiva que posibilita "emancipar un nombre de sus referencias conceptuales unívocas" (Laclau, 2005, p. 140), para lograr constituir un nuevo sentido a partir de una articulación equivalencial de diferentes elementos en uno solo. Esta es la función catacrética, función que permite comprender el pueblo "como otra cosa distinta del lugar al que vienen a depositarse y superponerse, como en una simple superficie de inscripción, unos objetos instaurados de antemano" (Foucault, 1979, p. 69). Este, por el contrario, debe ser entendido como "una práctica social a través de la cual se constituyen los objetos y sujetos de los que se habla" (Bolívar, 2006, p. 24).

Segundo, al ser entendido el pueblo como el resultado de una práctica hegemónica llevada a cabo por sujetos ubicados histórica, social, política, económica y culturalmente dentro de un contexto histórico y social particular de producción, circulación y apropiación, el contexto, como categoría de análisis, adquiere total importancia. Como muy bien lo advierte Bourdieu (1999), si se examina toda producción discursiva fuera de su contexto de producción, circulación y apropiación, es decir, como una producción autónoma, objetiva y desinteresada, se pierde de vista la importancia de las condiciones de posibilidad que, precisamente, posibilitan que dicha producción adquiera cierta eficacia como "palabra creadora, que confiere vida a lo que anuncia" (p. 16). Dicho en otras palabras, al olvidar el contexto de producción, circulación y apropiación como categoría de análisis, se pierde de vista la capacidad del discurso para organizar y clasificar la realidad social, de acuerdo a las condiciones de posibilidad que su mismo contexto social de producción le permite. En este punto, compartimos la advertencia realizada por Grossberg (2010), respecto a que ninguna práctica cultural (textos y discurso incluido) existe "al margen de las fuerzas y de las relaciones del contexto que las constituyen como tal" (p. 95), ya que estas son justamente, el resultado de las condiciones sociales e históricas, que posibilitan que dichas prácticas adquieran cierta significatividad.

Por último, podríamos señalar que el pueblo, al ser entendido en una de sus dimensiones como el resultado de una práctica discursiva, a través de la cual se produce un conjunto específico de formas de entender, organizar, clasificar y valorar a dicho pueblo, también debe ser entendido y abordado como un sujeto político por medio del cual se produce su propio proceso de identificación. Así, el pueblo es el resultado de un efecto performativo de constituir al propio pueblo como un pueblo conservador, católico, liberal, colombiano, venezolano, ecuatoriano, radical, cristiano, etc.

\section{Conclusiones}

Para cerrar esta reflexión acerca de la teoría del populismo elaborada por Laclau, es primordial reseñar los siguientes aspectos. En primer lugar, reconocer su esfuerzo teórico constante, continuo y riguroso por restituir lo político como elemento fundante de lo social. Como se abordó a lo largo del texto, no existe una esencia de lo social que determine la forma como los sujetos y 
sus relaciones se deban llevar a cabo. Por el contrario, corresponden a rearticulaciones hegemónicas, donde la única garantía que existe es que, precisamente, no existe garantía alguna de que las cosas ocurran de una manera determinada. No obstante, esto no quiere decir que exista una total indeterminación en la forma como se organizan las relaciones sociales, sino que, como lo sostiene el propio Laclau, corresponden a un cierre arbitrario del exceso de sentido de lo social por medio del cual se hegemoniza una parte de este.

En segundo lugar, esta desencialización de lo social nos conduce a un elemento fundamental de la perspectiva de Laclau: el "rescate" del populismo de la posición marginal, a la cual fue confinado y condenado por las ciencias sociales de corte racionalista y mecanicista. Como lo señala Laclau, el populismo es una de las formas políticas por medio de las cuales se instituye un determinado orden social de manera hegemónica, esto es, el populismo no es una lógica irracional ni mecanicista, sino una lógica hegemónica a través de la cual se articulan, en una cadena equivalencial, una pluralidad de demandas y sujetos particulares produciendo como resultado un nuevo sujeto político que intenta representar dichas demandas y sujetos. Este nuevo sujeto es el pueblo.

Por último, la consecuencia de esta operación, es que el pueblo comienza a significar lo que aquellos que lo estructuran "eligen" que signifique, esto nos lleva a dos posibles escenarios de articulación respecto al populismo. Por un lado, un proceso articulatorio donde confluyen grupos y demandas con un carácter democrático radical, teniendo como propósito u objetivo la desestructuración de las relaciones de dominación y subordinación presentes en un contexto particular. Este sería el escenario de deseabilidad para el fortalecimiento de una democracia radical, tal y como la desarrollan Laclau y Mouffe (1985). Pero, por otro lado, este proceso también puede tomar otra dirección. La articulación de un movimiento reaccionario a través del cual se mantenga o reproduzca el estatus quo. La dinámica que se logre imponer como hegemónica determinará qué tipo de pueblo se logró constituir y qué tipo de contenidos son los que defiende.

Esta forma a través de la cual se produce el pueblo, nos dirige a reafirmar el llamado de atención realizado por Laclau y Mouffe respecto a la manera cómo los grupos sociales subordinados deben llevar a cabo sus luchas políticas:

Si las demandas de un grupo subordinado se presentan como demandas puramente negativas y subversivas de un cierto orden - estrategia de oposición-, sin estar ligadas a ningún proyecto viable de reconstrucción de un nuevo orden - estrategia de construcción de un nuevo orden-, su capacidad de actuar hegemónicamente estará excluida desde el comienzo. (p. 235)

En la capacidad de plantear, planear y llevar a la práctica una estrategia de construcción de un nuevo orden, es donde radica el reto de los nuevos movimientos populistas que logren surgir como alternativas al orden social hegemónico. Esta tarea es la que esperamos que logren cumplir con éxito. 


\section{REFERENCIAS}

Austin, J. L. (1962/1971). ¿Cómo hacer cosas con palabras? Palabras y acciones. Barcelona: Paidós.

Bolívar, I. (2006). Discursos emocionales y experiencias de la política: Las FARC y las AUC en los procesos de negociación del conflicto (1998-2005). Tesis de grado para optar el título de Magister en Antropología Social. Universidad de los Andes, Facultad de Ciencias Sociales, Departamento de Antropología, Bogotá.

Bourdieu, P. (1999). ¿Qué significa hablar? Economía de los intercambios lingüísticos. Madrid: Ediciones Akal.

Bowman, G. (2005). Violencia constitutiva e imaginario nacionalista: la construcción del 'pueblo' en Palestina y la 'ex Yugoslavia'. En F. Panizza (Comp), El populismo como espejo de la democracia (pp 161-199). Buenos Aires: Fondo de Cultura Económica.

Foucault, M. (1970/1979). La Arqueología del Saber. México: Siglo Veintiuno Editores S.A.

Grossberg, L. (2004). Entre consenso y hegemonía: notas sobre la forma hegemónica de la política moderna. Revista de humanidades Tabula Rasa, 2, 49-57.

Grossberg, L. (2010) Estudios culturales: Teoría, política y práctica. Valencia: Letra Capital.

Hall, S. (2010) Sin garantías. Trayectorias y problemáticas en los estudios culturales (E. Restrepo, C. Walsh, \& V. Vich, Eds). Instituto de estudios sociales y culturales Pensar, Universidad Javeriana. Instituto de Estudios Peruanos. Universidad Andina Simón Bolívar, sede Ecuador: Envión Editores.

Jäger, S. (2001/2003). Discurso y conocimiento: aspectos teóricos y metodológicos de la crítica del discurso y del análisis de dispositivos. En M. Meyer, \& R. Wodak (Comps), Métodos de análisis crítico del discurso (pp 61-100). Barcelona: Gedisa S.A. Jociles, M. (2005). El análisis del discurso: de cómo utilizar desde la antropología social la propuesta analítica de Jesús Ibáñez. Ava. Revista de Antropología, (7), 147-167.

Laclau, E, \& Chantal, M. (1985). Hegemonía y estrategia socialista. Hacia una radicalización de la democracia. Argentina: Fondo de cultura Económica.

Laclau, E. (1977). Política e ideología en la teoría marxista. Capitalismo, fascismo, populismo. México D.F.: Siglo Veintiuno Editores s.a.

Laclau, E.. (2005). La razón populista. Buenos Aires: Fondo de Cultura Económica.

Laclau, E.. (2005a). Populismo. ¿Qué nos dice el nombre? En F. Panizza (Comp.), El populismo como espejo de la democracia (pp 51-70). Buenos Aires: Fondo de Cultura Económica.

Laycock, D. (2005). Populismo y nueva derecha en el Canadá inglés. En F. Panizza (Comp), El populismo como espejo de la democracia (pp 241-282). Buenos Aires: Fondo de Cultura Económica.

Lowndes, J. (2005). De la violencia fundacional a la hegemonía política: el populismo conservador de George Wallace. En F. Panizza (Comp.), El populismo como espejo de la democracia (pp 201240). Buenos Aires: Fondo de Cultura Económica.

Mouffe, C. (2005). El 'fin de la política' y el desafío del populismo de derecha. En F. Panizza (Comp.). El populismo como espejo de la democracia (pp 71-96). Buenos Aires: Fondo de Cultura Económica.

Mouffe, C. (2007). En torno a lo político. Buenos Aires: Fondo de Cultura Económica. 
Panizza, F (Comp). (2005). El populismo como espejo de la democracia. Buenos Aires: Fondo de Cultura Económica.

Perelman, C. (1977/1997). El imperio retórico. Retórica y argumentación. Bogotá D.C.: Norma S.A.
Reyes, O. (2005). Conservadurismo skinhead: un proyecto populista fallido. En F. Panizza (Comp.), El populismo como espejo de la democracia (pp 133-159). Buenos Aires: Fondo de Cultura Económica. 